\title{
Anti-Neutrophil Cytoplasmic Antibody Vasculitis in Pediatric Patients: Is the Incidence Rising?*
}

\author{
Christine Sethna ${ }^{1}$, Rachel Frank ${ }^{1}$, Lulette Infante ${ }^{1}$, Beth Gottlieb ${ }^{2}$, Anne Eberhard ${ }^{2}$, \\ Xiaotong Wang ${ }^{3}$, Howard Trachtman ${ }^{4 \prime}$ \\ ${ }^{1}$ Department of Pediatrics, Division of Nephrology, Cohen Children's Medical Center of New York, New York, USA \\ ${ }^{2}$ Department of Pediatrics, Division of Rheumatology, Cohen Children's Medical Center of New York, New York, USA \\ ${ }^{3}$ Department of Pathology, North Shore University Hospital of the North Shore-LIJ Health System, New York, USA \\ ${ }^{4}$ Department of Pediatrics, Division of Nephrology, NYU Langone Medical Center, New York, USA \\ Email: " howard.trachtman@nyumc.org
}

Received March 24, 2012; revised April 25, 2012; accepted May 21, 2012

\begin{abstract}
Objectives: Anti-neutrophil cytoplasmic antibody (ANCA)-associated vasculitis is an autoimmune disease usually seen in middle-aged and older adults but which is rare in children and adolescents. We sought to determine if there has been a change in the incidence of this disorder. Methods: Single-center, retrospective review. Results: Over the last 2 years, we have encountered a striking increase in the frequency of this disease in pediatric patients. All eight patients seen during this period had renal involvement and 5 patients rapidly progressed to end stage kidney disease. The prognosis was worse in younger patients, those with microscopic polyangiitis, and those with chronic kidney damage in the diagnostic renal biopsy. Conclusions: We report these observations to highlight this change in the epidemiology of ANCA-associated vasculitis and to promote earlier recognition and treatment of this severe form of glomerulonephritis.
\end{abstract}

Keywords: Anti-Neutrophil Cytoplasmic Antibody (ANCA); Vasculitis; Epidemiology

\section{Introduction}

Anti-neutrophil cytoplasmic antibody (ANCA)-associated vasculitis is an autoimmune disorder that usually presents with sudden onset disease. The organ systems that are primarily affected include the kidney and lung [1]. There are two main subtypes based on the identity of the causative antibody - granulomatosis with polyangiitis (GPA) [Wegener's] and microscopic polyangiitis (MPA). The former is associated with elevated titers of antibody to proteinase 3 and is characterized by cytoplasmic staining of neutrophils (cANCA) while the later is marked by antibodies to myeloperoxidase that stain neutrophils in a perinuclear pattern (pANCA) [1,2]. The kidney injury is characterized by necrotizing vasculitis and glomerular inflammation with minimal immune complex deposition. GPA and MPA require timely recognition and initiation of induction therapy in order to prevent permanent organ damage [2].

Both subtypes of ANCA-associated disease are rare and generally occur in middle-aged and elderly patients. Although it does occur in children, in most large patient

\footnotetext{
"Financial Disclosures: None. No payment of any type was made to anyone to produce the manuscript. Conflict of Interest Declarations: None.

${ }^{\#}$ Corresponding author.
}

series, less than $10 \%$ of all cases occur in the pediatric age range [3]. Over the last 2 years we have encountered an unexpected surge in the number of cases of ANCAassociated disease, well above the occurrence of at most one case annually at our referral center. We report our experience in order to promote heightened awareness of this condition in children in the hope that earlier case definition and prompt implementation of effective treatments will lead to improved clinical outcomes.

\section{Patients and Methods}

Patients were identified by a review of a clinical database maintained in the Division of Nephrology with cross referencing with the Division of Rheumatology to ensure inclusion of all cases. Out-patient charts and hospital records were retrieved and reviewed and the following data were recorded: age at diagnosis, gender, race and ethnicity, presenting symptoms and physical findings, $\mathrm{CBC}$, serum BUN, creatinine, cholesterol, albumin, C3, and $\mathrm{C} 4$ levels, ANCA and ANA titers, and urinary protein excretion expressed as the protein:creatinine ratio (mg:mg) in a first morning urine sample. The kidney biopsy findings were described including the active and chronic changes in the kidney tissue. The renal functional 
status at baseline and the last follow-up visit and the induction and maintenance treatment regimens were tabulated. The retrospective chart review was approved by the North Shore-LIJ Institutional Review Board.

\section{Results}

The demographic features, treatment and clinical outcomes of our patients are summarized in Table 1. The diagnosis was confirmed both serologically and by renal histopathology in all of the cases. Five patients had MPA and 3 (all male) had GPA (formerly called Wegener's). There were no children with Churg-Strauss disease. All three patients with GPA had pulmonary involvement. In one case, there was acute pulmonary hemorrhage that prompted urgent initiation of plasmapheresis. There was evidence of concomitant gastrointestinal disease in one child, central nervous system involvement in 2, dermatological findings in 2, and cardiac manifestations (noninfectious intraventricular mass) in 1 patient. None of the patients had documented sinusitis, subglottic stenosis, or nasal deformity (saddle nose).

All patients received intravenous "pulse" methylprednisolone, 7 were given intravenous cyclophosphamide, 3 rituximab, and 3 were treated with plasmapheresis. The later three cases were characterized by severe renal insufficiency and the need for dialysis at the time of presentation.

Maintenance therapy included oral prednisone with mycophenolate mofetil or methotrexate. The outcomes for kidney function were poor. All five patients with MPA progressed to end stage kidney disease (ESKD); 2 are on chronic dialysis and 3 received a kidney transplant. Allograft function is normal without recurrent disease in the later subgroup. Two patients on maintenance medications have normal kidney function and 1 is still receiving induction therapy and steadily improving.

The number of cases in this series is limited. However, reviewing our recent experience with ANCA-associated disease, it would appear that pediatric patients with GPA tend to be older ( $\geq 16$ years of age) and to have a better prognosis for preservation of kidney compared to those with MPA. In addition, most of the patients displayed active glomerular inflammation and their clinical outcomes ranged from development of ESKD to recovery of kidney function. In contrast, three patients had evidence of chronic kidney injury with glomerulosclerosis, interstitial fibrosis, and tubular atrophy in the diagnostic biopsy that was done at the time of presentation and they all required renal replacement therapy.

\section{Discussion}

The diagnosis of ANCA-associated vasculitis and nephritis was confirmed in all 8 cases based on positive serological test results and the presence of characteristic histopathological findings in the kidney biopsy tissue. Misclassification of the disease is unlikely in view of the normal C3, ANA and double-stranded DNA antibody titers in all cases and the lack of alternative pathology findings that would suggest a different glomerular disorder. The only cause of glomerulonephritis that might resemble MPA/GPA in children and adolescents is Henoch Schonlein purpura nephritis; however, this diagnosis is excluded by the absence of mesangial IgA deposition in all patients [4].

In most previous reports of ANCA-associated vasculitis, less than $10 \%$ of cases are documented in childhood [3]. There is one previous report from Canada that, similar to our case series, describes a rising incidence of ANCA-associated disease and which suggests that it is a generalized phenomenon [5]. The diagnostic criteria for the disease are equally valid in children and adults, implying that atypical signs and symptoms at presentation are unlikely to explain the lower incidence that has been reported in childhood (6). Serological testing for ANCA

Table 1. Clinical features of pediatric patients with ANCA-associated nephritis.

\begin{tabular}{ccccccccc}
\hline Pt \# & $\begin{array}{c}\text { Age (yr) at } \\
\text { Onset/Gender }\end{array}$ & Ethnicity & Disease Type & $\begin{array}{c}\text { Glomerular } \\
\text { Lesions, Active }\end{array}$ & $\begin{array}{c}\text { Glomerular } \\
\text { Lesions, Chronic }\end{array}$ & $\begin{array}{c}\text { Interstitial } \\
\text { Fibrosis/Tubular Atrophy }\end{array}$ & Treatments & Outcome \\
\hline 1 & $6 \mathrm{~F}$ & W & MPA & N/A & N/A & N/A & S, C & Transplant \\
2 & $12 \mathrm{~F}$ & H & MPA & 100 & 0 & 0 & S, C & Transplant \\
3 & $6 \mathrm{M}$ & H & MPA & $10 \%-21 \%$ & $80 \%-90 \%$ & $20 \%-30 \%$ & S, C & Dialysis (Peritoneal) \\
4 & $12 \mathrm{~F}$ & W & MPA & 0 & $60 \%$ & $20 \%$ & S, C & Transplant \\
5 & $18 \mathrm{M}$ & W & GPA & $90 \%-100 \%$ & 0 & 0 & S, C, R, PE & Scr 1.39, eGFR 95 \\
6 & $16 \mathrm{M}$ & B & GPA & $26 \%-32 \%$ & 0 & 0 & S, C, & Scr 1.27, eGFR 98 \\
7 & $12 \mathrm{~F}$ & B & MPA & $23 \%-37 \%$ & $50 \%-60 \%$ & $30 \%-40 \%$ & S, C, R, PE & Dialysis (Hemo) \\
8 & $16 \mathrm{M}$ & W & GPA & $67 \%-73 \%$ & 0 & 0 & S, R, PE & Scr 2.56, eGFR 49 \\
\hline
\end{tabular}

The numbers in the columns detailing the pathology findings indicate the percentage of glomeruli affected by the abnormality or the interstitial area displaying the specific lesion. MPA: microscopic polyangiitis; GPA: granulomatosis with polyangiitis; S: solumedrol; C: cyclophosphamide; R: rituximab; PE: plasmapheresis. 
may occasionally be negative in children and a positive result is not required for diagnosis. Therefore, a kidney biopsy may be required to confirm pauciimmune glomerulonephritis with vasculitic or necrotizing lesions [7]. All of our cases had characteristic features of ANCA-associated disease and positive serology. The trend shows no evidence of abating and a new case was encountered during the preparation of this report. The patient was not included in this series because she was 21 years of age and would be considered an adult even though she was cared for by pediatric subspecialists. Thus, we propose that the increased incidence encountered over the last 2 years reflects a true change in the frequency of this complex illness in pediatric patients. At present, the cause for the sudden change in the epidemiology of ANCA-associated disease remains a mystery.

Recent studies suggest that ANCA may develop due to molecular mimicry with fimbrial protein antigens in bacteria [8]. Although there is a follow-up report that has shed some doubt on this association [9], it raises the possibility that altered environmental exposure may account for the surge in pediatric cases of ANCA-associated disease over the last few years.

ANCA-associated vasculitis is usually abrupt and severe in onset and requires early identification to achieve optimal outcomes. The present cases, several of whom had irreversible kidney damage at the time of diagnosis, may heighten awareness of ANCA-associated vasculitis in pediatric patients so that serological testing is performed promptly and aggressive therapy is administered in a timely manner. It is worth noting that our outcomes for kidney function were much worse than in a series of 8 children with ANCA-associated vasculitis (7 GPA, 1 MPA), of whom only 1 developed ESKD over a follow-up period ranging from $11-30$ years [10]. This suggests that both the incidence and severity of ANCA-associated vasculitis in childhood may be changing. A younger age, the diagnosis of MPA, and the presence of chronic glomerular and interstitial damage appear to be indicators of a poor prognosis for maintenance of normal kidney function. However, children who have significant active glomerular inflammation can respond to therapy with a return of renal function. The logistical difficulties in implementing effective but costly therapies such as plasmapheresis and rituximab that are required to achieve rapid remission underscore the urgency of establishing the diagnosis of GPA/MPA in an accurate and timely manner [11-13].

In conclusion, ANCA-associated vasculitis is not a new entity, but a change in epidemiology of this severe illness warrants concern among physicians who care for individual patients and public health officials. We encourage the formation of a registry through established societies in pediatric nephrology and rheumatology to monitor the incidence and outcomes of patients with these rare diseases.

\section{REFERENCES}

[1] R. J. Falk and J. C. Charles Jennette, "ANCA Disease: Where Is This Field Heading?" Journal of the American Society of Nephrology, Vol. 21, No. 5, 2010, pp. 745-752. doi:10.1681/ASN.2009121238

[2] A. E. Berden, F. Ferrario, E. Christian Hagen, D. R. Jayne, J. Charles Jennette, K. Joh, I. Neumann, L. H. Noël, C. D. Pusey, R. Waldherr, J. A. Bruijn and I. M. Bajema, "Histopathologic Classification of ANCA-Associated Glomerulonephritis," Journal of the American Society of $\mathrm{Ne}$ phrology, Vol. 21, No. 10, 2010, pp. 1628-1636. doi:10.1681/ASN.2010050477

[3] C. E. Rodrigues, M. R. Callado, C. A. Nobre, F. E. Moura, R. M. Vieira, L. A. de Albuquerque and W. P. Vieira, "Wegener's Granulomatosis: Prevalence of the Initial Clinical Manifestations-Report of Six Cases and Review of the Literature," Revista Brasileira de Reumatologia, Vol. 50, No. 2, 2010, pp. 150-164. doi:10.1590/S0482-50042010000200005

[4] R. Coppo, P. Cirina, A. Amore, R. A. Sinico, A. Radice and C. Rollino, "Properties of Circulating IgA Molecules in Henoch Schonlein Purpura Nephritis with Focus on Neutrophil Cytoplasmic Antigen IgA Binding (IgAANCA): New Insight into a Debated Issue. Italian Group of Renal Immunopathology Collaborative Study on Henoch Schonlein Purpura in Adults and Children," $\mathrm{Ne}$ phrology Dialysis Transplantation, Vol. 12, No. 11, 1997, pp. 2269-2276. doi:10.1093/ndt/12.11.2269

[5] S. Grisaru, G. W. Yuen, P. M. Miettunen and L. A. Hamiwka, "Incidence of Wegener's Granulomatosis in Children," The Journal of Rheumatology, Vol. 37, No. 2, 2010, pp. 440-442. doi:10.3899/jrheum.090688

[6] D. A. Cabral, A. G. Uribe, S. Benseler, K. M. O’Neil, P. J. Hashkes, G. Higgins, A. S. Zeft, D. J. Lovell, D. J. Kingsbury, A. Stevens, et al., "Classification, Presentation, and Initial Treatment of Wegener's Granulomatosis in Childhood," Arthritis Rheum, Vol. 60, No. 11, 2009, pp. 34133424. doi:10.1002/art.24876

[7] M. Shimizu, T. Sekiguchi, et al., "A Case of a 6-Year Old Girl with Anti-Neutrophil Cytoplasmic Antibody- Negative Pauci-Immune Crescentic Glomerulonephritis," Clinical and Experimental Nephrology, Vol. 15, No. 4, 2011, pp. 596-601. doi:10.1007/s10157-011-0423-3

[8] R. Kain, M. Exner, R. Brandes, et al., "Molecular Mimicry in Pauci-Immune Focal Necrotizing Glomerulonephritis," Nature Medicine, Vol. 14, No. 10, 2008, pp. 1088-1096. doi:10.1038/nm.1874

[9] A. J. Roth, M. C. Brown, R. N. Smith, A. K. Badwar, et al., "Anti-LAMP-2 Antibodies Are Not Prevalent in Patients with Antineutrophil Cytoplasmic Autoantibody Glomerulonephritis," Journal of the American Society of Nephrology, Vol. 23, No. 5, 2012, pp. 545-555.

[10] N. Arulkumaran, S. Jawad, S.W. Smith, L. Harper, P. Brogan, C. D. Pusey and A. D. Salama, "Long-Term Outcome of Paediatric Patients with ANA Vasculitis," $P e$ - 
diatric Rheumatology Online Journal, Vol. 9, 2011, p. 12. doi:10.1186/1546-0096-9-12

[11] A. Casian and D. Jayne, "Plasma Exchange in the Treatment of Wegener's Granulomatosis, Microscopic Polyangiitis, Churg-Strauss Syndrome and Renal Limited Vasculitis," Current Opinion in Rheumatology, Vol. 23, No. 1, 2011, pp. 12-17. doi:10.1097/BOR.0b013e32834120c1

[12] R. B. Jones, J. Willem Cohen Tervaert, T. Hauser, R. Luqmani, M. D. Morgan, C. A. Peh, et al., "Rituxamab
Versus Cyclophosphamide in ANCA-Associated Renal Vasculitis," The New England Journal of Medicine, Vol. 363, 2010, pp. 211-220. doi:10.1056/NEJMoa0909169

[13] J. H. Stone, P. A. Merkel, et al., "Rituximab Versus Cyclophosphamide for ANCA-Associated Vasculitis," The New England Journal of Medicine, Vol. 363, 2010, pp. 221-232. doi:10.1056/NEJMoa0909905

\section{Abbreviations}

ANA, anti-nuclear antibody

ANCA, Anti-neutrophil cytoplasmic antibody

cANCA, cytoplasmic ANCA

pANCA, perinuclear ANCA

BUN, blood urea nitrogen

C3, C4, complement component 3, 4

CBC, complete blood count

ESKD, end stage kidney disease

GPA, granulomatosis with polyangiitis [Wegener's]

MPA, microscopic polyangiitis 